\title{
The construction of a two-dimensional reproducing kernel function and its application in a biomedical model
}

\author{
Qi Guo ${ }^{\mathrm{a}, *}$ and Shu-Ting Shen ${ }^{\mathrm{b}}$ \\ ${ }^{a}$ Department of Mathematics, Harbin Institute of Technology, Harbin, Heilongjiang, China \\ ${ }^{\mathrm{b}}$ Health Science Center, Peking University, Beijing, China
}

\begin{abstract}
.
BACKGROUND: There are two major classes of cardiac tissue models: the ionic model and the FitzHugh-Nagumo model. During computer simulation, each model entails solving a system of complex ordinary differential equations and a partial differential equation with non-flux boundary conditions. The reproducing kernel method possesses significant applications in solving partial differential equations. The derivative of the reproducing kernel function is a wavelet function, which has local properties and sensitivities to singularity. Therefore, study on the application of reproducing kernel would be advantageous. OBJECTIVE: Applying new mathematical theory to the numerical solution of the ventricular muscle model so as to improve its precision in comparison with other methods at present.

METHODS: A two-dimensional reproducing kernel function inspace is constructed and applied in computing the solution of two-dimensional cardiac tissue model by means of the difference method through time and the reproducing kernel method through space.

RESULTS: Compared with other methods, this method holds several advantages such as high accuracy in computing solutions, insensitivity to different time steps and a slow propagation speed of error. It is suitable for disorderly scattered node systems without meshing, and can arbitrarily change the location and density of the solution on different time layers.

CONCLUSIONS: The reproducing kernel method has higher solution accuracy and stability in the solutions of the twodimensional cardiac tissue model.
\end{abstract}

Keywords: Reproducing kernel, two-dimensional, cardiac tissue model, compact finite difference

\section{Introduction}

With the rapid development of computer technology, virtual reality techniques have been applied in many fields, including the virtual heart in biomedical engineering [1,2].

Simulation research on the human heart began decades ago. This research has resulted in the creation of mathematical model simulations of the major cardiac tissue cells of various species, as well as the production of biochemical models and the heart-produced electricity model [3-5].

In China, virtual heart research is still in its infancy [6-8]. The First Military Medical University and Zhejiang University have conducted many preliminary studies in this field. For example, they analyzed

\footnotetext{
${ }^{*}$ Corresponding author: Qi Guo, Department of Mathematics, Harbin Institute of Technology, Harbin 150001, Heilongjiang, China. Tel.: +86 18946192559; E-mail: guoqi@hit.edu.cn.
}

0928-7329/16/\$35.00 (c) 2016 - IOS Press and the authors. All rights reserved This article is published online with Open Access and distributed under the terms of the Creative Commons Attribution NonCommercial License. 
the simulation results of a number of abnormal electrocardiograms according to the structure of the cardiac electrophysiology model and the ECG algorithm, which is based on the single-cell action potential calculation. Zhejiang University built a mechanical model of the left cardiac ventricle using the finite element method that is based on the structure of cardiac ventricle tissue fiber, and was compared with MR imaging of the magnetic marker to obtain preliminary simulation results.

Cardiac tissue conduction models and computer simulation studies have transformed heart recognition from a qualitative to quantitative analysis. Previously, there have been two major classes of cardiac tissue models: ionic models and FitzHugh-Nagumo models (FHN). Regardless of the model used for computer simulation, it is necessary to solve a system of complex ordinary differential equations (ODE) and a partial differential equation (PDE) with non-flux boundary conditions.

The reproducing kernel method has important applications for solving partial differential equations [9, 10]. Because the derivative of the reproducing kernel function is the wavelet function at the same time, and the wavelet function has the local property as well sensitivity to singularity. The reproducing kernel method has the following advantages compared to the common finite difference method:

(1) The accuracy of the solution is higher and is not sensitive to the time step. Additionally, the error accumulation rate is slow.

(2) It can be applied to a disorderly scattered node system without meshing.

(3) There are no meshing problems in actual calculations, so the position and density of the solution can be arbitrarily changed at different times in the calculation according to the specific characteristics of the problems, giving them greater flexibility and applicability.

We study the application of reproducing kernels in the numerical solutions of a cardiac tissue model, apply new mathematical theory to solve the numerical solution of the ventricular muscle model with high accuracy, and the method of solving the ventricular muscle model is the difference method and the finite element method at present, but it has no new breakthrough in solving precision. The purpose of this paper is break through the existing numerical solution and improve the solution accuracy. However, the reproducing kernel method is complicated and has a slow operation speed that requires high computer configuration, and the current computer configuration can meet the requirements of the standard. Therefore, it will play a role in promoting the study of the virtual heart model if we can improve the accuracy of this level and can have a further study in the physiological activities of the heart. Because a single cell simulation requires the calculation of dozens of complex mathematical equations involving thousands of state variables, hundreds of thousands, or even millions, of these cells are required to build a simulation model of a virtual heart. The requirements for storage space and processing are large. So, as to further solve the problems of the current virtual human heart model, which is far from realistic, and the reproduction of all physiological activities in vivo to make some contribution.

In this article, we construct a reproducing kernel function in $W_{2}$ space and use it to solve the twodimensional cardiac tissue model. Further, using the FitzHugh-Nagumo model as an example, we discuss the method based on reproducing kernel theory that is used to solve the two-dimensional cardiac tissue model, by use of the difference method through time, and the reproducing kernel method through space. In the simulation experiment, we compared the reproducing kernel method and the compact finite difference method [6] at solving the same partial differential equation and found that the reproducing kernel method is more precise. 


\section{Construction of two-dimensional reproducing kernel}

\subsection{Definition of $W_{2}$ space}

Definition Suppose $f(x, y)$ is a real continuous function on the rectangular region $D=[a, b] \times[c, d]$. If for any $\varepsilon>0$, there exists a $\delta>0$, such that for any group of nonintersecting subregions $\left\{D_{i}=\left[a_{i}, b_{i}\right] \times\left[c_{i}, d_{i}\right]\right\}(i=1,2, \cdots, n)$ of $D$, there is $\sum_{i=1}^{n}\left|f\left(D_{i}\right)\right|<\varepsilon$, so long as the sum of area $\sum_{i=1}^{n}\left|D_{n}\right|<\delta$, in which

$$
f\left(D_{i}\right) \stackrel{\text { def }}{=} f\left(b_{i}, d_{i}\right)-f\left(b_{i}, c_{i}\right)-f\left(a_{i}, d_{i}\right)+f\left(a_{i}, c_{i}\right)
$$

and $f(x, c), f(a, y)$ are all one-dimensional completely continuous functions. Then we define $f(x, y)$ as a bivariate completely continuous function on $D$.

Denote

$$
W_{2}=\left\{u_{x, y} \mid \frac{\partial u}{\partial x}, \frac{\partial u}{\partial y}\right\}
$$

are completely continuous functions on $D$,

$$
\left.\frac{\partial^{\alpha} u}{\partial x^{\alpha_{1}} \partial y^{\alpha_{2}}} \in L^{2}(D), \alpha=\alpha_{1}+\alpha_{2}, \alpha_{i}=0,1,2, i=1,2\right\}
$$

where $D$ is the domain of the bivariate function in $W_{2}$. For convenience, we can take $D=[0,1] \times[0,1]$. Then, the inner product of $W_{2}$ is as follows:

$$
\begin{aligned}
(u, v) & =\iint_{D}\left(u v+2 \frac{\partial u}{\partial x} \frac{\partial v}{\partial x}+2 \frac{\partial u}{\partial y} \frac{\partial v}{\partial y}+4 \frac{\partial^{2} u}{\partial x \partial y} \frac{\partial^{2} v}{\partial x \partial y}+\frac{\partial^{2} u}{\partial x^{2}} \frac{\partial^{2} v}{\partial x^{2}}+\frac{\partial^{2} u}{\partial y^{2}} \frac{\partial^{2} v}{\partial y^{2}}\right. \\
& \left.+2 \frac{\partial^{3} u}{\partial x^{2} \partial y} \frac{\partial^{3} v}{\partial x^{2} \partial y}+2 \frac{\partial^{3} u}{\partial x \partial y^{2}} \frac{\partial^{3} v}{\partial x \partial y^{2}}+\frac{\partial^{4} u}{\partial x^{2} \partial y^{2}} \frac{\partial^{4} v}{\partial x^{2} \partial y^{2}}\right) d x d y
\end{aligned}
$$

Denote norm as $\|\cdot\|=(\cdot, \cdot)^{\frac{1}{2}}$.

\section{2. $W_{2}$ Spatial reproducing kernel function}

Let $R_{M}\left(M^{\prime}\right)=R_{x}(\xi) R_{y}(\eta), M=M(x, y), M^{\prime}=M^{\prime}(\xi, \eta)$ and

$$
R_{s}(t)=\left\{\begin{array}{l}
{\left[a_{1}(s)+a_{2}(s) t\right] e^{t}+\left[a_{3}(s)+a_{4}(s) t\right] e^{-t}, t \leqslant s} \\
{\left[b_{1}(s)+b_{2}(s) t\right] e^{t}+\left[b_{3}(s)+b_{4}(s) t\right] e^{-t}, t>s}
\end{array}\right.
$$

be the reproducing kernel function of the reproducing kernel space $W_{2}^{2}[0,1]$,

where

$$
\begin{aligned}
& a_{1}(s)=b_{1}(s)+\frac{1}{4}(s+1) e^{-s}, a_{2}(s)=b_{2}(s)-\frac{1}{4} e^{-s} \\
& a_{3}(s)=b_{3}(s)+\frac{1}{4}(s-1) e^{s}, a_{4}(s)=b_{4}(s)-\frac{1}{4} e^{s} \\
& b_{1}(s)=\frac{3}{2 \Delta}\left[\left(9-5 e^{-2}\right) e^{s}+\left(e^{-2}-3\right) s e^{s}+\left(7-3 e^{-2}\right) e^{-s}+\left(5-3 e^{-2}\right) s e^{-s}\right]
\end{aligned}
$$




$$
\begin{aligned}
& b_{2}(s)=\frac{1}{\Delta}\left[\frac{3}{2}\left(e^{-2}-3\right) e^{s}+3\left(1-e^{-2}\right) s e^{s}+\left(\frac{9}{2} e^{-2}-\frac{11}{2}\right) e^{-s}+2 s e^{-s}\right] \\
& b_{3}(s)=\frac{1}{2 \Delta}\left[\left(9 e^{2}-1\right) e^{s}+\left(11-9 e^{-2}\right) s e^{s}+15\left(e^{-2}-1\right) e^{-s}+3\left(e^{2}+1\right) s e^{-s}\right] \\
& b_{4}(s)=\frac{1}{\Delta}\left[\left(\frac{9}{2} e^{2}-\frac{7}{2}\right) e^{s}+2 s e^{s}+\frac{3}{2}\left(e^{2}+1\right) e^{-s}+3\left(e^{2}-1\right) s e^{-s}\right] \\
& \Delta=36 \operatorname{ch} 2-44
\end{aligned}
$$

Next we will prove that $R_{M}\left(M^{\prime}\right)$ is the $W_{2}$ spatial reproducing kernel function.

Proof: $R_{x y}(a, \eta), R_{x y}(\xi, b)$ are completely continuous functions with $\xi, \eta$ respectively, and because they are one-dimensional reproducing kernels, we know:

$$
R_{x y}\left(D_{i}\right)=\left(R_{x}\left(b_{i}\right)-R_{x}\left(a_{i}\right)\right)\left(R_{y}\left(d_{i}\right)-R_{y}\left(c_{i}\right)\right) \leqslant\left(b_{i}-a_{i}\right)\left(d_{i}-c_{i}\right)=\left|D_{i}\right| .
$$

So $R_{x y}(\xi, \eta)$ is a bivariate continuous function on $D$ when $x, y$ is fixed as a function of $\xi, \eta$. If $\frac{\partial}{\partial \xi} R_{x y}(\xi, \eta), \frac{\partial}{\partial \eta} R_{x y}(\xi, \eta), \frac{\partial^{\alpha} u}{\partial x^{\alpha_{1}} \partial y^{\alpha_{2}}} \in L^{2}(D)\left(\alpha=\alpha_{1}+\alpha_{2}, \alpha_{i}=0,1,2, i=1,2\right)$, then $R_{x y}(\xi, \eta) \in$ $W_{2}(D)$.

$\forall u \in W_{2}$, so that we have

$$
\begin{aligned}
\left(u(\cdot, \cdot), R_{x y}(\cdot, \cdot)\right) & =\int_{c}^{d} \int_{a}^{b}\left\{u(\xi, \eta) R_{x}(\xi) R_{y}(\eta)+2 u_{\xi}^{\prime}(\xi, \eta) \frac{\partial}{\partial \xi} R_{x}(\xi) R_{y}(\eta)\right. \\
& +2 u_{\eta}^{\prime}(\xi, \eta) R_{x}(\xi) \frac{\partial}{\partial \eta} R_{y}(\eta)+4 u_{\xi \eta}^{\prime \prime}(\xi, \eta) \frac{\partial}{\partial \xi} R_{x}(\xi) \frac{\partial}{\partial \eta} R_{y}(\eta) \\
& +u_{\xi^{2}}^{\prime \prime}(\xi, \eta) \frac{\partial^{2}}{\partial \xi^{2}} R_{x}(\xi) R_{y}(\eta)+u_{\eta^{2}}^{\prime \prime}(\xi, \eta) R_{x}(\xi) \frac{\partial^{2}}{\partial \eta^{2}} R_{y}(\eta) \\
& +2 \frac{\partial^{3} u(\xi, \eta)}{\partial \xi^{2} \partial \eta} \frac{\partial^{2}}{\partial \xi^{2}} R_{x}(\xi) \frac{\partial}{\partial \eta} R_{y}(\eta)+2 \frac{\partial^{3} u(\xi, \eta)}{\partial \xi \partial \eta^{2}} \frac{\partial}{\partial \xi} R_{x}(\xi) \frac{\partial^{2}}{\partial \eta^{2}} R_{y}(\eta) \\
& +\frac{\partial^{4} u(\xi, \eta)}{\partial \xi^{2} \partial \eta^{2}} \frac{\partial^{2}}{\partial \xi^{2}} R_{x}(\xi) \frac{\partial^{2}}{\partial \eta^{2}} R_{y}(\eta) \\
& =\int_{c}^{d} \int_{a}^{b} R_{y}(\eta)\left(u(\xi, \eta) R_{x}(\xi)+2 u_{\xi}^{\prime}(\xi, \eta) \frac{\partial}{\partial \xi}+u_{\xi^{2}}^{\prime \prime}(\xi, \eta) \frac{\partial^{2}}{\partial \xi^{2}} R_{x}(\xi)\right) \\
& +\frac{\partial}{\partial \eta} R_{y}(\eta)\left(2 u_{\eta}^{\prime}(\xi, \eta) R_{x}(\xi)+4 u_{\xi \eta}^{\prime \prime}(\xi, \eta) \frac{\partial}{\partial \xi} R_{x}(\xi)+2 \frac{\partial^{3} u(\xi, \eta)}{\partial \xi^{2} \partial \eta} \frac{\partial^{2}}{\partial \xi^{2}} R_{x}(\xi)\right) \\
& +\frac{\partial^{2}}{\partial \eta^{2}} R_{y}(\eta)\left(u_{\eta^{2}}^{\prime \prime}(\xi, \eta) R_{x}(\xi)+2 \frac{\partial^{3} u(\xi, \eta)}{\partial \xi \partial \eta^{2}} \frac{\partial}{\partial \xi} R_{x}(\xi)+\frac{\partial^{4} u(\xi, \eta)}{\partial \xi^{2} \partial \eta^{2}} \frac{\partial^{2}}{\partial \xi^{2}} R_{x}(\xi)\right) \\
& =\int_{c}^{d}\left|u(x, \eta) R_{y}(\eta)+2 u_{\eta}^{\prime}(x, \eta) \frac{\partial}{\partial \eta} R_{y}(\eta)+u_{\eta^{2}}^{\prime \prime}(x, \eta) \frac{\partial^{2}}{\partial \eta^{2}} R_{y}(\eta)\right| d \eta=u(x, y)
\end{aligned}
$$

That is to say $R_{x y}(\xi, \eta)$ is the reproducing kernel in $W_{2}(D)$.

Suppose $\left\{M_{i}\right\}_{1}^{\infty}=\left\{\left(x_{i}, y_{i}\right)\right\}_{1}^{\infty}$ is a row of mutually different irregular arranged pitch points on $D$, denoted $\varphi_{i}(M)=R_{M_{i}}(M), i=1,2, \cdots$. From $R_{M_{i}}(M)$ 's expression, we know that $\left\{\varphi_{i}(M)\right\}_{1}^{\infty}$ is a 
linearly independent group, so we can make it the Schmidt standard orthogonal:

$$
\varphi_{i}^{*}(M)=\sum_{j=1}^{i} a_{j i} \varphi_{j}(M), a_{i i}>0, i=1,2, \cdots
$$

Theorem $\mathbf{1} W_{2}$ space is Hilbert.

Theorem $2\left\{\varphi_{i}^{*}(M)\right\}_{1}^{\infty}$ is the complete norm orthogonal system of $W_{2}$ space.

\section{Computing the solution of two-dimensional cardiac muscle model with reproducing kernel}

The FitzHugh-Nagumo model can be described by partial differential equations that are defined on $D=\left[x_{\min }, x_{\max }\right] \times\left[y_{\min }, y_{\max }\right]:$

$$
\left\{\begin{array}{l}
\frac{d u}{d t}=\frac{u-u^{3} / 3-v}{\varepsilon}+\frac{\partial^{2} v}{\partial x^{2}}+\frac{\partial^{2} v}{\partial y^{2}}+S t i \\
\frac{d v}{d t}=\varepsilon(u+b-\gamma v)
\end{array}\right.
$$

where $u$ is the cross membrane voltage, $v$ is restore variable, $S t i$ is a shock at $x=x_{\min }$ when $t=0$, and $\varepsilon, b, \gamma$ are constants. Using the non-flux boundary condition $\left.\frac{\partial V}{\partial x}\right|_{\substack{x=x_{\max } \\ x=x_{\min }}}=\left.\frac{\partial V}{\partial y}\right|_{\substack{y=y_{\max } \\ y=y_{\min }}}=0$, we over on the difference to Eq. (5) in the time first:

$$
\begin{aligned}
& u^{n+1}=u^{n}+\frac{\Delta t}{\varepsilon}\left(u^{n}-\left(u^{n}\right)^{3} / 3-v^{n}\right)+\Delta t\left(\frac{\partial^{2} u^{n}}{\partial x^{2}}+\frac{\partial^{2} u^{n}}{\partial y^{2}}\right) \\
& v^{n+1}=v^{n}+\Delta t \varepsilon\left(u^{n}+b-\gamma v^{n}\right)
\end{aligned}
$$

Let

$$
u^{n}=\sum_{k=1}^{N} c_{k}^{n} \varphi_{k}(M)
$$

Where the reproducing kernel $\varphi_{k}(M)$ is determined by Eq. (3).

Equation (6) can be written as

$$
\begin{aligned}
& u^{n+1}(M)=u^{n}(M)+\frac{\Delta t}{\varepsilon}\left(u^{n}(M)-\left(u^{n}(M)\right)^{3} / 3-v^{n}(M)\right)+\Delta t \sum_{k=1}^{N} c_{k} \mu_{k}(M) \\
& \alpha^{n}(M)=u^{n}(M)+\frac{\Delta t}{\varepsilon}\left(u^{n}(M)-\left(u^{n}(M)\right)^{3} / 3-v^{n}(M)\right) \\
& \beta^{n}(M)=\sum_{k=1}^{N} c_{k} \mu_{k}(M)
\end{aligned}
$$

Through simplification, Eq. (9) can be written as

$$
u^{n+1}(M)=\alpha^{n}(M)+\Delta t \beta^{n}(M)
$$


If we set the value to above formula at each pitch point $\left\{M_{i}\right\}_{1}^{N}$, and write in matrix forms, we get:

$$
\left[\begin{array}{l}
u^{n+1}\left(M_{1}\right) \\
u^{n+1}\left(M_{2}\right) \\
\vdots \\
u^{n+1}\left(M_{N}\right)
\end{array}\right]=\left[\begin{array}{l}
\alpha^{n}\left(M_{1}\right) \\
\alpha^{n}\left(M_{2}\right) \\
\vdots \\
\alpha^{n}\left(M_{N}\right)
\end{array}\right]+\Delta t\left[\begin{array}{l}
\beta^{n}\left(M_{1}\right) \\
\beta^{n}\left(M_{2}\right) \\
\vdots \\
\beta^{n}\left(M_{N}\right)
\end{array}\right]
$$

Written simply as:

$$
U^{n+1}=A^{n}+\Delta t B^{n}
$$

From Eq. (10), we know $A^{n}$ can be expressed by $U^{n}$ and $V^{n}$, where

$$
V^{n}=\left[v^{n}\left(M_{1}\right) v^{n}\left(M_{2}\right) \cdots v^{n}\left(M_{N}\right)\right]^{\prime}
$$

And from Eq. (8), we know that

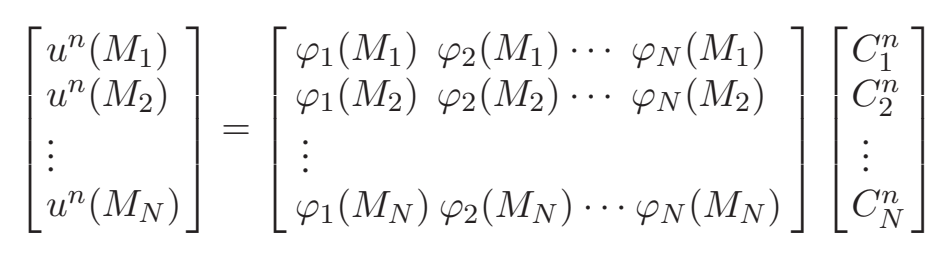

can be written as

$$
U^{n}=K C^{n}
$$

Because $K^{-1}$ exists from the linearly independent property of reproducing kernel function $\left\{\varphi_{i}(M)\right\}_{1}^{N}$ and from $U^{n}$ we get $C^{n}=K^{-1} U^{n}$, we can obtain $B^{n}$ from Eq. (11), and the following iteration is generated:

\section{Algorithm 3.1:}

1) From the initial condition $U^{0}, V^{0}$ and Eqs (10), (14), (7), we can obtain $A^{0}, C^{0}, V^{1}$;

2) From Eq. (11) and $C^{0}$, we can get $B^{0}$;

3) From Eq. (13) and $A^{0}, B^{0}$ we can get $U^{1}$;

4) By analogizing, we can get $U^{n}, V^{n}$.

\section{Simulation experiment}

We conducted simulation experiments to compare the precision levels between the reproducing kernel method and the compact finite difference method [6] for solving partial differential equations.

1) First, we compare the reproducing kernel method and the compact finite difference method for solving the two-dimensional heat conductivity equation, which is similar to the FitzHugh-Nagumo model and has a real solution.

2) Second, we use the algorithm given in Section 3, which solves the FitzHugh-Nagumo model with the reproducing kernel method, to carry the solution to it. 
Example 1. Solve the heat conductivity equation

For $\frac{\partial V}{\partial t}=\frac{\partial^{2} V}{\partial x^{2}}+\frac{\partial^{2} V}{\partial y^{2}},(x, y) \in D=[0,1] \times[0,1], t \in[0, T], T=0.001$, the initial condition is: $\left.V\right|_{t=0}=V^{0}=10 \cos (\pi x) \cos (\pi y)$, and the boundary condition is $\left.\frac{\partial V}{\partial x}\right|_{\substack{x=0 \\ x=1}}=\left.\frac{\partial V}{\partial y}\right|_{\substack{y=0 \\ y=1}}=$ 0 , its real solution is $V=10 * \exp \left(-2 \pi^{2} t\right) * \cos (\pi x) * \cos (\pi y)$.

Solution: using the algorithm introduced in Section 3, carry on the difference to the time, we have

$$
V^{n+1}=V^{n}+\Delta t\left(\frac{\partial^{2} V^{n}}{\partial x^{2}}+\frac{\partial^{2} V^{n}}{\partial y^{2}}\right)
$$

Let

$$
V^{n}=\sum_{k=1}^{N} C_{k}^{n} \varphi_{k}(M)
$$

where $N$ is the number of the selected discrete points, and the again-nucleation $\varphi_{k}(M)$ is determined by Eq. (3).

That is $\varphi_{k}(M)=R_{M_{k}}(M)=R\left(M_{k}, M\right), M=M(x, y), M_{k}=M_{k}\left(x_{i}, y_{j}\right),(i, j$ are determined by $M_{k}$ ).

From the definition of the two-dimensional reproducing kernel in $W_{2}$ space, we know $R_{M_{k}}(M)=$ $R_{x_{i}}(x) R_{y_{j}}(y)$.

If we substitute Eq. (16) into Eq. (15), we have

$$
\begin{aligned}
\sum_{k=1}^{N} C_{k}^{n+1} \varphi_{k}(M) & =\sum_{k=1}^{N} C_{k}^{n} \varphi_{k}(M)+\Delta t \sum_{k=1}^{N} C_{k}^{n}\left(R_{x_{i}}^{\prime \prime}(x) R_{y_{j}}(y)+R_{x_{i}}(x) R_{y_{j}}^{\prime \prime}(y)\right) \\
& =\sum_{k=1}^{N} C_{k}^{n}\left[\varphi_{k}(M)+\Delta t \mu_{k}(M)\right]
\end{aligned}
$$

where $\mu_{k}(M)=R_{x_{i}}^{\prime \prime}(x) R_{y_{j}}(y)+R_{x_{i}}(x) R_{y_{j}}^{\prime \prime}(y)$

Value the above formula at each pitch point $\left\{M_{i}\right\}_{1}^{N}$, and write in matrix form

$$
\begin{aligned}
& {\left[\begin{array}{cccc}
\varphi_{1}\left(M_{1}\right) & \varphi_{2}\left(M_{1}\right) & \cdots & \varphi_{N}\left(M_{1}\right) \\
\varphi_{1}\left(M_{2}\right) & \varphi_{2}\left(M_{2}\right) & \cdots & \varphi_{N}\left(M_{2}\right) \\
\vdots & & & \\
\varphi_{1}\left(M_{N}\right) & \varphi_{2}\left(M_{N}\right) & \cdots & \varphi_{N}\left(M_{N}\right)
\end{array}\right]\left[\begin{array}{l}
C_{1}^{n+1} \\
C_{2}^{n+1} \\
\vdots \\
C_{N}^{n+1}
\end{array}\right]=} \\
& {\left[\begin{array}{cccc}
\varphi_{1}\left(M_{1}\right)+\Delta t \mu_{1}\left(M_{1}\right) & \varphi_{2}\left(M_{1}\right)+\Delta t \mu_{2}\left(M_{1}\right) & \cdots & \varphi_{N}\left(M_{1}\right)+\Delta t \mu_{N}\left(M_{1}\right) \\
\varphi_{1}\left(M_{2}\right)+\Delta t \mu_{1}\left(M_{2}\right) & \varphi_{2}\left(M_{2}\right)+\Delta t \mu_{2}\left(M_{1}\right) & \cdots & \varphi_{N}\left(M_{2}\right)+\Delta t \mu_{N}\left(M_{2}\right) \\
\vdots & & & \\
\varphi_{1}\left(M_{N}\right)+\Delta t \mu_{1}\left(M_{N}\right) & \varphi_{2}\left(M_{N}\right)+\Delta t \mu_{2}\left(M_{N}\right) & \cdots & \varphi_{N}\left(M_{N}\right)+\Delta t \mu_{N}\left(M_{2}\right)
\end{array}\right]\left[\begin{array}{l}
C_{1}^{n} \\
C_{2}^{n} \\
\vdots \\
C_{N}^{n}
\end{array}\right]}
\end{aligned}
$$

The above formula can be written as $A C^{n+1}=B C^{n}$.

If

$$
\left[V^{k}\right]=\left(V^{k}\left(M_{1}\right), V^{k}\left(M_{1}\right), \cdots, V^{k}\left(M_{N}\right)\right)^{\prime}, \text { then }\left[V^{n+1}\right]=A C^{n+1},\left[V^{n+1}\right]=B C^{n}
$$

Because of the linearly independent property of the function $\left\{\varphi_{i}(M)\right\}_{1}^{N}$ we know that $A^{-1}$ exists, from the initial condition we can get $C^{0}=A^{-1}\left[V^{0}\right]$, and by carrying the iteration to Eq. (17), we can get $V^{n}$. 
Table 1

Comparison of error between the reproducing kernel method and the finite difference method

\begin{tabular}{lcc}
\hline Number of iterations & Reproducing kernel error & Finite difference error \\
\hline$n=20$ & 0.00750 & 0.01813 \\
$n=40$ & 0.01063 & 0.03563 \\
$n=60$ & 0.01001 & 0.05438 \\
$n=80$ & 0.00754 & 0.07125 \\
$n=100$ & 0.00313 & 0.08813 \\
\hline
\end{tabular}

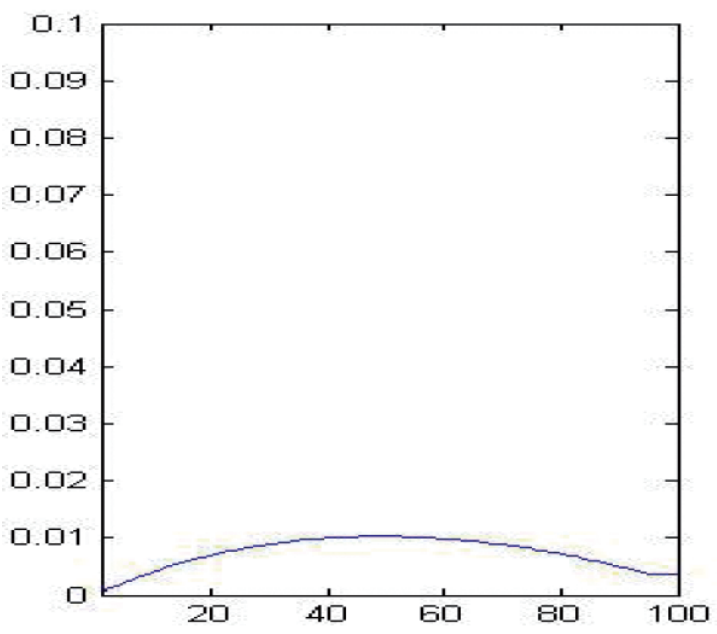

Fig. 1. Reproducing kernel method error.

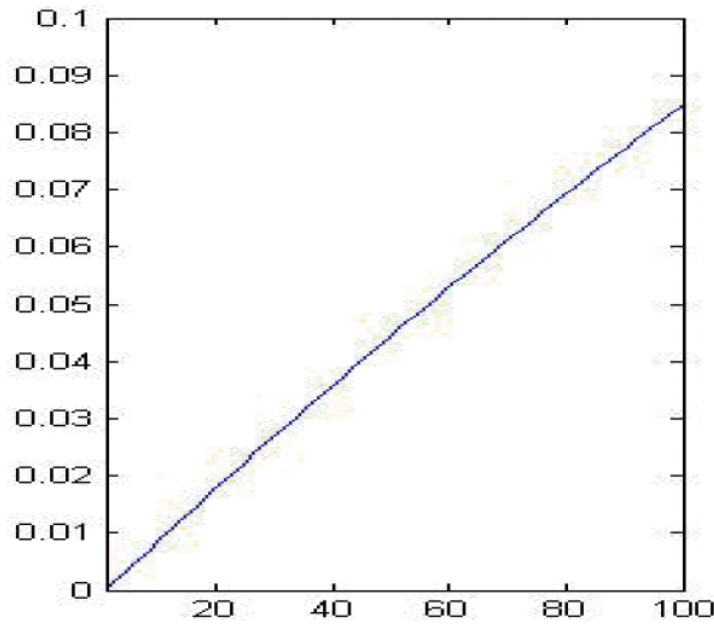

Fig. 2. Finite difference method errors.

Program the realization with Matlab, and take the time length $\Delta t=10^{-5}$. For the iteration numbers of time $n(1 \leqslant n \leqslant 100)$, output the biggest error in these interpolations points (Fig. 1). In order to obtain the superiority of the reproducing kernel method, we analyze and compare the errors about reproducing kernel method and the finite difference method. The error of finite difference method shown in Fig. 2. The error comparison between reproducing kernel method and the finite difference method is shown in Table 1.

The error is defined as $\max \left|e_{i}\right|$, where $e_{i}$ is difference between the true solution and the numerical solution, and $i$ is the order number of the selected discrete points in $W_{2}$ space.

Note: the horizontal coordinates of the above two figures are the number of iterations of time, and the vertical coordinates are the maximum errors of these interpolation nodes at each time layer.

From the above results, we can see that the reproducing kernel method has higher precision and stability.

Example 2. Computing the solution of the partial differential system of equations (FitzHugh-Nagumo model):

$$
\left\{\begin{array}{l}
\frac{d u}{d t}=\frac{u-u^{3} / 3-v}{\varepsilon}+\frac{\partial^{2} v}{\partial x^{2}}+\frac{\partial^{2} v}{\partial y^{2}}+S t i \\
\frac{d v}{d t}=\varepsilon(u+b-\gamma v)
\end{array}\right.
$$

Where $D=\left[x_{\min }, x_{\max }\right] \times\left[y_{\min }, y_{\max }\right]$ is the domain of definition, $u$ is the cross membrane voltage, $v$ is the restore variable, Sti is a shock at $x=x_{\min }$ when $t=0$, and $\varepsilon, b, \gamma$ are constants. We use the nonflux boundary condition: $\left.\frac{\partial V}{\partial x}\right|_{\substack{x=x_{\max } \\ x=x_{\min }}}=\left.\frac{\partial V}{\partial y}\right|_{\substack{y=y_{\max } \\ y=y_{\min }}}=0$, and let $\varepsilon=0.1, b=1.0, \gamma=0.5$, Sti $=5.8$. 


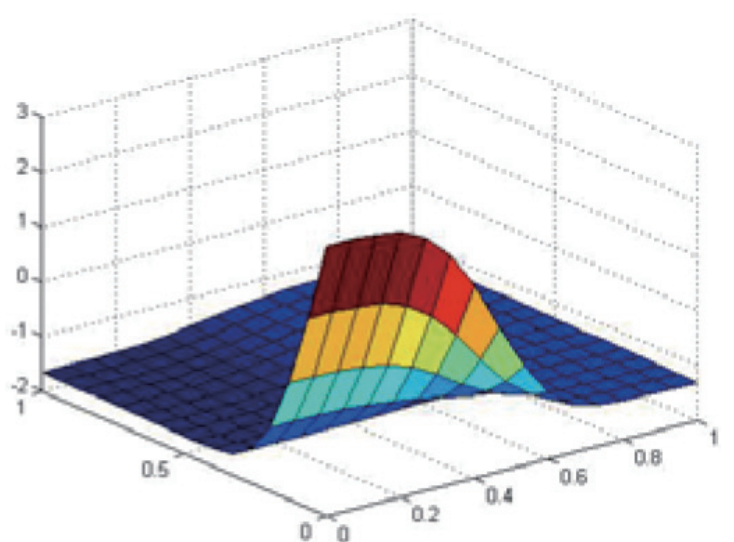

Fig. 3. When $t=10 \mathrm{~ms} u$-graph (reproducing kernel method).

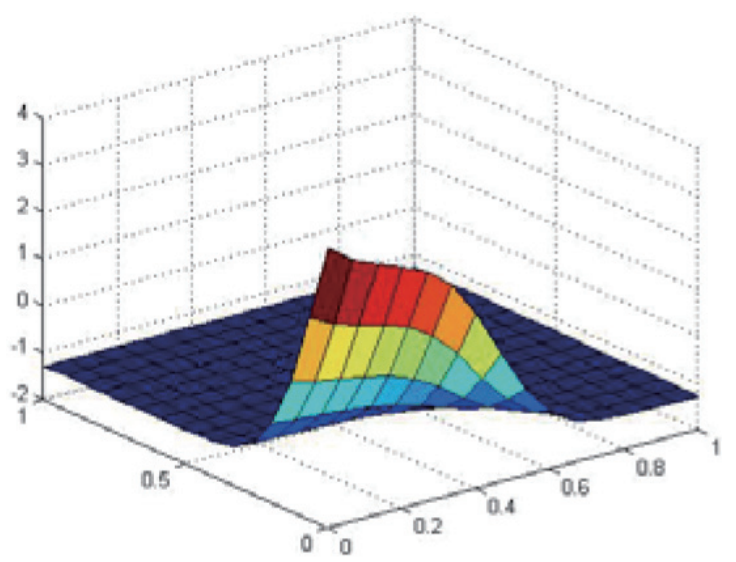

Fig. 5. When $t=10 \mathrm{~ms}, u$-graph (finite difference method).

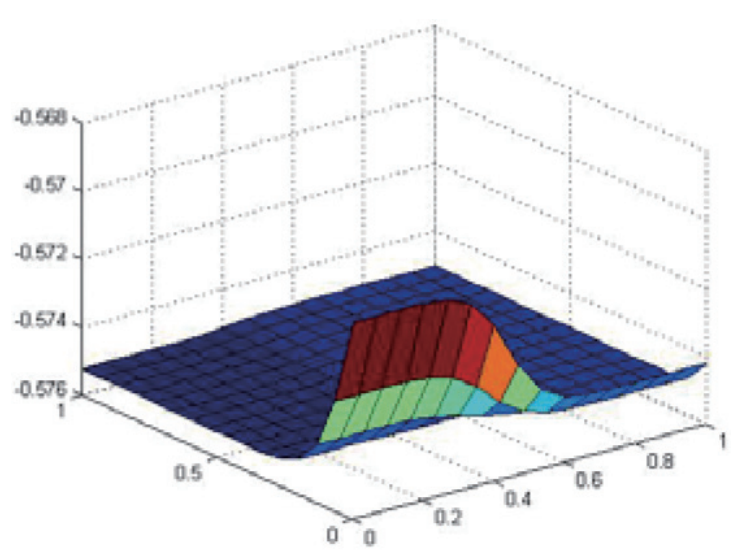

Fig. 4. When $t=10 \mathrm{~ms} v$-graph (reproducing kernel method).

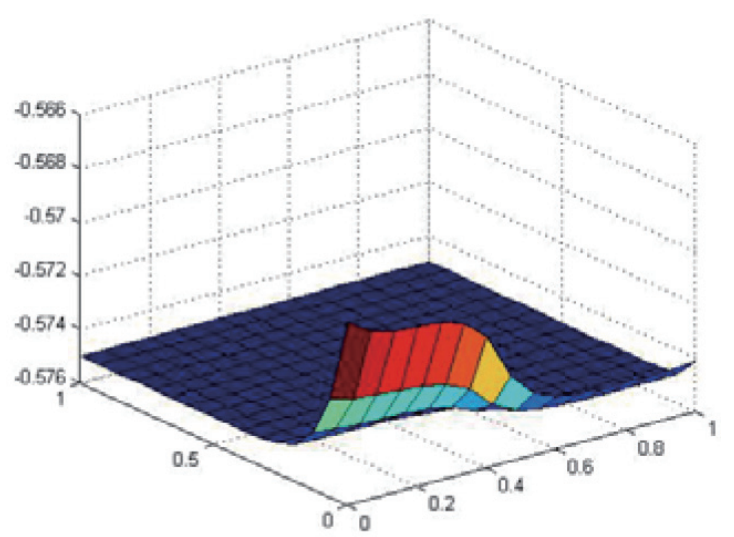

Fig. 6. When $t=10 \mathrm{~ms}$, v-graph (finite difference method).

Solution: We will use the algorithm 3.1 introduced in Section 3.

Figures 3 and 4 show the $u$ and $v$ when $t=10 \mathrm{~ms}$ under the reproducing kernel method. In order to make a comparison, we also use the finite difference method to solve the system of Eq. (18) (Figs 5-6).

\section{Discussion}

The results indicated that, compared to the finite difference method provided by article [6], when the reproducing kernel method is applied in solving the same Fitzhugh Nagumo model, discrepancies are evident in variable $u$, whereas variable $\mathrm{v}$ exhibits no detectable differences. However, as shown in Example 1, the reproducing kernel method can produce a higher level of accuracy and stability as the number of iterations increases. 


\section{Conclusion}

A two-dimensional reproducing kernel function in space was constructed and used to compute the solution of a two-dimensional cardiac tissue model. Using the FitzHugh-Nagumo model as an example, we have discussed the method based on reproducing kernel theory to solve the two-dimensional cardiac tissue model by use of difference method through time and the reproducing kernel method through space. Using simulations, we compared the reproducing kernel method and the compact finite difference method [6] for solving the same partial differential equation. The results show that the reproducing kernel method has higher precision and stability.

\section{Acknowledgement}

This work is supported by Heilongjiang Natural Science foundation (No. GFQQ2440501411).

\section{References}

[1] Palladino, Joseph L, Zukus, Ryan L. Left ventricular model parameters and cardiac rate variability. Proceedings of the Annual International Conference of the IEEE Engineering in Medicine and Biology Society, EMBS, pp. 6817-6820, 2011, 33rd Annual International Conference of the IEEE Engineering in Medicine and Biology Society, EMBS 2011.

[2] Zhong Liang, Su Boyang, Zhang JunMei, et al. FSI simulation of intra-ventricular flow in patient-specific ventricular model with both mitral and aortic valves. Proceedings of the Annual International Conference of the IEEE Engineering in Medicine and Biology Society, EMBS, pp. 703-706, 2013, 2013 35th Annual International Conference of the IEEE Engineering in Medicine and Biology Society, EMBC 2013.

[3] Su Boyang, Zhong Liang, Wang XiKun. Numerical simulation of patient-specific left ventricular model with both mitral and aortic valves by FSI approach. Computer Methods and Programs in Biomedicine, 2014; 113(2): 474-482.

[4] Gohean, Jeffrey R, George, Mitchell J, Pate, Thomas D. Verification of a computational cardiovascular system model comparing the hemodynamics of a continuous flow to a synchronous valveless pulsatile flow left ventricular assist device. ASAIO Journal, 2013; 59(2): 107-116.

[5] Roy CP, Kerckhoffs, Sarah N Healy. Computational methods for cardiac electromechanics. proceedings of the IEEE, April, 2006; 94(4): 769-779.

[6] Jichao Zhao, Yinbin Jin, Li Ma. A highly efficient and accurate algorithm for solving the partial differential equation in cardiac tissue models. Proceedings of the 2006 WSEAS International Conference on Mathematical Biology and Ecology, Miami, Florida, USA, January 18-20, 2006; pp. 81-86.

[7] Bernus O, Verschelde H, Panfilov AV. Modified ionic models of cardiac tissue for efficient large scale computations. Phys. Med. Biol, 2002; 47: 1947-1959.

[8] Jin YB, Yang L, Zhang H, Kuo YH, Huang YC. Numerical algorithm for conduction of action potential in twodimensional cardiac ventricle tissue. Journal of Xi' an Jiaotong University, 2004; 38(8): 851-854.

[9] Guo Qi, Yuan Ai-ling, Hong Bing-rong. The Reproducing Kernel Method to Solve Motion Trojectory for the Space Aircraft to Track an Goal. Journal of Astronautics, 2006; 27: 145-150, Sup.

[10] Cui Ming-gen, Wu Bo-ying. Reproducing kernel space numerical analysis. Science Press, China, 2004: 1-110. 